Research Article

\title{
Post graduate survey and evaluation of role of animal experimentation, status and relevance of current syllabus, and challenges for alignment with requirements of the pharmaceutical industry
}

\author{
Prashant Dass $^{1}$, Pradeep Javedar $^{2}$, Rajat Mishra ${ }^{3}$, Michell Gulabani ${ }^{4}$
}

\begin{abstract}
${ }^{1}$ Scientific Project Manager, Abbott India Limited, Mumbai, India

${ }^{2}$ Drug Safety Physician, Quintiles, Bengaluru, India ${ }^{3}$ Department of Pharmacology, AIIMS, Patna, Bihar, India ${ }^{4}$ Department of Anesthesiology, Dr. R.M.L. Hospital, New Delhi, India
\end{abstract}

Received: 16 March 2016

Revised: 01 May 2016

Accepted: 10 May 2016

*Correspondence to:

Dr. Prashant Dass,

Email: prashantdass@ymail.com

Copyright: () the author(s), publisher and licensee Medip Academy. This is an openaccess article distributed under the terms of the Creative Commons Attribution NonCommercial License, which permits unrestricted noncommercial use, distribution, and reproduction in any medium, provided the original work is properly cited.

\begin{abstract}
Background: The current scenario has brought the validity of animal experimentation (AE) under scrutiny. The requirements from an academician in the pharmaceutical industry are not sufficiently handled. A gnawing need was felt to conduct a study amongst the post graduate MD pharmacologists regarding the role of $\mathrm{AE}$, relevance of current syllabus and challenges faced in the pharmaceutical industry.

Methods: This questionnaire-based survey was carried out amongst post graduate $(\mathrm{PG})$ residents pursuing MD pharmacology. Students were enrolled via social contacts, email, and in 2 workshops/conferences. The survey questionnaire consisted of 20 questions in total. 47 completed questionnaires were returned from a total 60 . The data was collected in an anonymous fashion to avoid bias.

Results: From the study we observe that PG residents were aware of the current $\mathrm{MCI} /$ University guidelines on AE. Only $42.5 \%(\mathrm{n}=20)$ of the volunteers replied that they had access to simulator software. Participants were of the opinion that the current PG teaching curriculum is ill-adapted to tackle challenges encountered in the pharmaceutical industry. Compulsory industry rotation $(31.9 \%)$ was one of the favoured suggested changes. Overall satisfaction with MD pharmacology was obtained in the positive.

Conclusions: We conclude that the importance of $\mathrm{AE}$ in pharmacology is irrefutable, although advent of alternatives is the direction to be headed in. The curriculum is archaic at times, thus warrants changes. Preparedness for pharmaceutical industry is also low and needs to be addressed.
\end{abstract}

Keywords: Post graduate survey, AE, Pharmaceutical industry, PG

\section{INTRODUCTION}

Pharmacology is one of the rapidly evolving fields in medical sciences. The current scenario in post graduate MD Pharmacology studies is quite different from even a decade before. The ever progressing field of pharmacology is becoming more and more tech-savvy and less dependent on age old tried and tested methods for post graduate (PG) teaching across medical colleges in India.
Animal experimentation (AE) has been a back bone of the basic life sciences and has been a fundamental in training of almost all pharmacology related post graduate courses. ${ }^{1}$ However, due to the perpetual legislatory changes in the current scenario, the validity and applicability of AE has come under intense scrutiny.

The changes ushered by the advent of numerous pharmaceutical and research based industries has led to a conscious re-thinking pertaining to the validity of PG 
curriculum. The Indian pharmaceutical industry is growing at a mind boggling pace, but unfortunately the post graduate syllabus has been found lacking many a times. Although topics such as research methodology, biostatistics, AE are adequately dealt with in the PG teaching program, 1 there are lacunae when we deal with fields of medical marketing, regulatory affairs, clinical pharmacology, intellectual property rights etc. ${ }^{2}$

A fresh PG MD Pharmacologist is a naïve entity whilst entering the pharmaceutical industry. It has been well documented that the current PG syllabus is static, inconsistent and incongruous with the demands of the pharmaceutical industry. ${ }^{3}$ Many authors agree that a constant need is felt to bring out changes in the PG training programmes in pharmacology, so that the students can acquire suitable skills for a particular job profile. $^{4}$

Dang et al, also brought to notice that a majority of MD Pharmacologists preferred to pursue a career in the pharmaceutical industry. ${ }^{4}$ The requirements from an academician in the teaching field are well understood, and the challenges faced are dealt head-on in the post graduate learning program. However, preparation for the hurdles and obstacles in the corporate sector are not sufficiently handled at this level.

Thus, a gnawing need was felt to conduct a questionnaire based survey amongst the post graduate MD pharmacology residents regarding the role of animal experimentation, relevance of current syllabus and challenges faced in the pharmaceutical industry.

\section{METHODS}

This questionnaire-based survey was carried out amongst PG residents pursuing MD pharmacology in various medical colleges across India. After due permission from institutional ethics committee, the study was conducted from October to December 2014. Various post graduate students were enrolled via social contacts, email, and in 2 workshops/conferences namely, advanced workshop on clinical pharmacology 2014 (AJIMS, Mangalore); annual conference of society of pharmacovigilance- SOPICON 2014 (JNMC-AMU, Aligarh).

The survey questionnaire consisted of 20 questions in total, 14 questions were to be rated on a scale of $0-10$ ( 0 minimum, 10 maximum and 5 being a neutral response), 3 questions had various options and required marking of 1 answer only, 2 questions were to be answered as yes or no; and 1 question was open ended requiring suggestion/input from the participating volunteers.

The questionnaire was provided to 60 post graduate MD Pharmacology residents from different medical colleges across India, from this, 47 completed questionnaires were returned to us for evaluation and appraisal. The data was collected and tabulated in an anonymous fashion to avoid any conflicts and bias. The survey questionnaire is attached in the appendix section of this research article.

\section{Statistical analysis}

MS-Excel 2010 was employed for statistical analysis. Data was presented with the aid of descriptive statistics including mean, standard deviation and percentages wherever appropriately applicable.

\section{RESULTS}

For the purpose of our study, 60 participants were initially provided with the survey questionnaire. Out of these 60 , only 47 fully filled forms were received by us during the course of this investigation. Only these 47 completed forms were further evaluated and analysed for the purpose of this study.

From the study we observe that PG pharmacology residents were more or less aware of the current $\mathrm{MCI} /$ University guidelines. The average response being $6.14 \pm 1.53$ (on a scale of $0-10$ ). This explicitly exhibits the fact that animal experimentation in pharmacology is a contentious topic and all concerned parties vow to be well aware of existing rules and guidelines.

According to (Figure 1) of our study, PG students opine that $\mathrm{AE}$ is more relevant in $\mathrm{PG}$ research/industry level $(6.34 \pm 2.73)$ when compared to UG level $(4.27 \pm 2.02)$ and PG academic level $(5.95 \pm 2.03)$. This reflects the view that standalone knowledge of $\mathrm{AE}$ is a must in the pharmaceutical industry, where research is of prime importance.

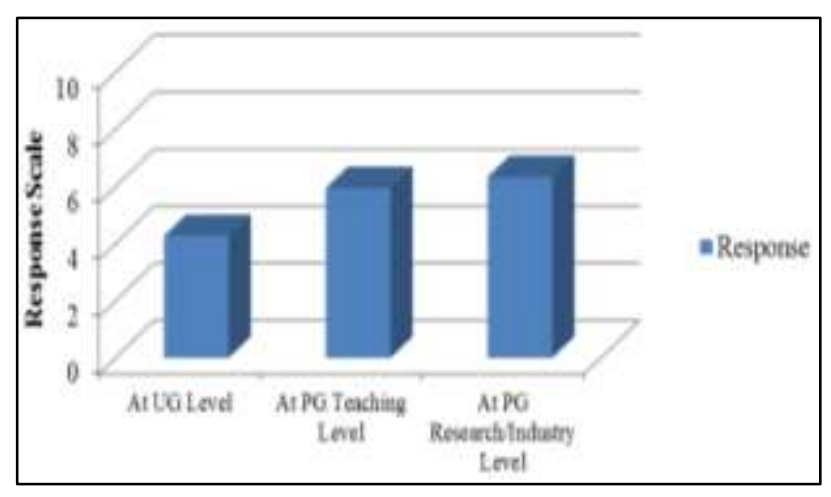

Figure 1: Relevance of experimental animals.

Subsequently, the participants were quizzed about their opinion on the type of $\mathrm{AE}$ which could be excluded from PG curriculum. A whopping majority of the replies were in the favour of removal of only those AE which involved animal sacrifice $(68.1 \%)$. A minority $(31.9 \%)$ of the participants wanted outright ouster of all forms of AE.

When asked about the knowledge of availability of alternative techniques to AE, we obtained a fairly neutral response $(5.08 \pm 2.32)$. This is one area in which our 
knowledge penetration can be expanded. With the demonstration of modern techniques in symposia, workshops etc., we can further our knowledge considerably regarding the comprehension of available alternatives to $\mathrm{AE}$.

As per expectations we received a fairly negative outlook when we enquired about the availability of these alternative techniques at the institution/college level (3.25 \pm 2.09$)$. We therefore observe and infer that we have a long road ahead of us as pharmacologists regarding provision of alternative methods to AE. Also, when enquired about the application of these techniques at the respective college/university level, we were again confounded with a fairly negative review (3.36 \pm 2.59 ). Thus we deduce that the overhaul of $\mathrm{AE}$ and available alternatives is to be undertaken at a large scale at a national level.

When asked to mention the currently available alternative techniques accessible at their institution/medical college, $42.5 \%(n=20)$ of the volunteers replied that they had access to simulator software or other such alternatives.

Next study question compares the different payment methods for obtaining the alternatives to AE. As per the replies we infer that an almost equal number of survey participants were in favour of government/university sponsorship $(41 \%)$, or a once payment method for available alternatives to animal experimentation (40\%). Probably because most of these alternative methods to AE are infrastructure intensive, most respondents were of the view of aided payment/once payment to lower the financial burden.

(Figure 2) summarises the opinion regarding preparation for teaching and pharmaceutical industry, provided by the current PG curriculum.

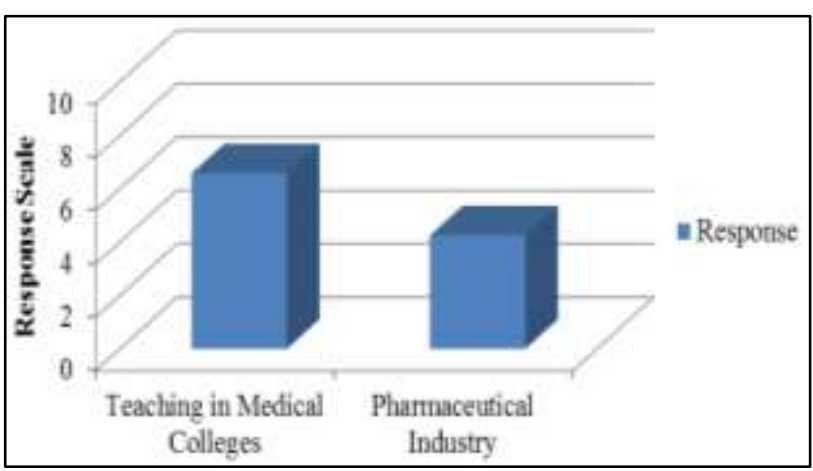

Figure 2: Equipment by PG curriculum for challenges ahead.

(Figure 2) clearly summarises that the participants are of the opinion that the current PG teaching curriculum equips them to tackle the knack for teaching in medical colleges well $(6.57 \pm 1.49)$. However, it presents a much grimmer picture when we consider the aid to tackle challenges encountered in the pharmaceutical industry (4.25 \pm 2.03$)$.

When asked about changes to be inculcated in the PG syllabus, the maximum response was garnered by compulsory industry rotations $(31.9 \%)$, followed by an additional emphasis on clinical pharmacology (29.3\%) (Figure 3).

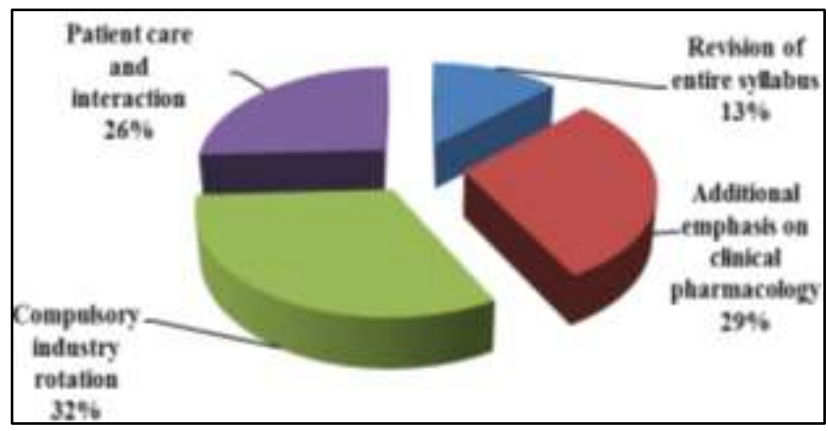

Figure 3: Changes to be inculcated in PG syllabus.

Clinical rotations were also considered a necessity and most of the volunteers exhibited a favourable outlook towards it $(6.74 \pm 1.68)$.

As per (Figure 4), we concur that seminars (7.21 \pm 1.70$)$ and clinical case discussions $(7.06 \pm 2.26)$ were deemed as the most effective learning tools in PG teaching of pharmacology. Experiments $(5.34 \pm 2.26)$ and short technique demonstrations $(4.51 \pm 2.45)$ brought up the rear amongst the choices.

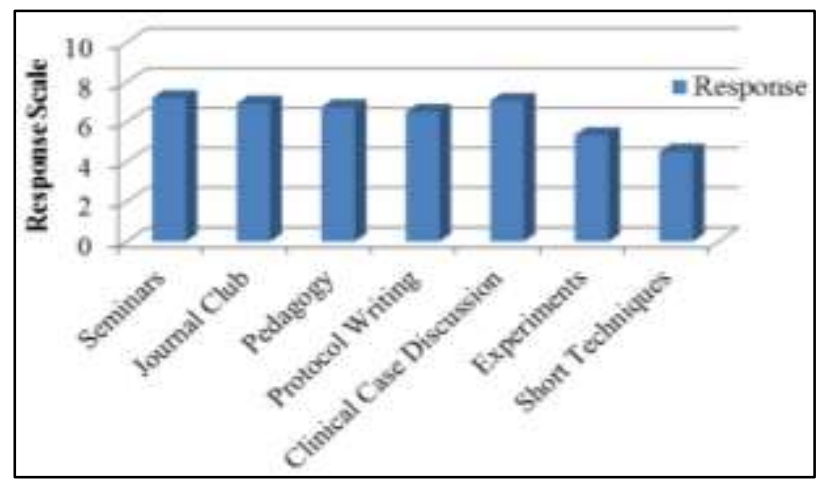

Figure 4: Teaching tools in PG curriculum.

A rather disturbing finding was the overall lack of encouragement towards research which was rated a mere 4+2.6. This reflects the lacklustre approach to good quality clinical research in medical college across India.

The subsequent interrogation explains the responses of the volunteers when enquired about their awareness about various responsibilities confronted in the pharmaceutical industry. From this a striking observation is inferred relating to the lack of awareness about the responsibilities as a regulator $(36.1 \%)$ and a researcher $(48.9 \%)$ in the 
pharmaceutical industry. The responsibilities of an employee as a clinical trial co-ordinator were supposedly well understood by the participants of this study (72.3\%).

For a candidate pursuing MD pharmacology, numerous add-ons are currently available to hone their skill levels so as to better adapt to the rigours of the pharmaceutical industry. As per (Figure 5) we infer that master's in business administration (MBA) held the least promise as an add-on tool to the armamentarium of the pharmacologist $(4.72 \pm 2.3)$.

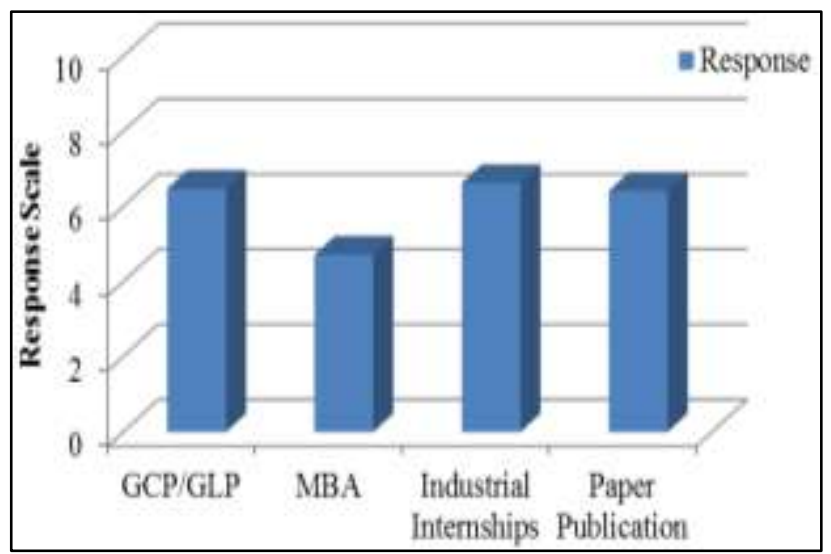

\section{Figure 5: Add-on skills as per usefulness for pharmaceutical industry.}

Another dismaying observation was that nearly half of the interviewees $(48.9 \%)$ were unaware and could not adequately differentiate their role and hierarchy in the pharmaceutical industry when compared with $\mathrm{PhD}$, Pharm D, M. Pharm etc.

When the participants were asked to evaluate the attraction of various sub-disciplines in the pharmaceutical industry, the most favoured was clinical trials $(7.06 \pm 1.67)$ while the least favoured of the lot was Bioavailability/Bioequivalence studies (5.21 1.41$)$.

The succeeding interrogation quizzed the participants to appraise the various national level workshops and conferences on their usefulness for preparation of $\mathrm{PG}$ residents for the pharmaceutical industry. The top honours went to Pharma tech by KEM, Mumbai $(7.06 \pm 2.28)$ and national workshop on clinical pharmacology and therapeutics by PGI, Chandigarh $(6.95 \pm 2.02)$, while IPSCON formed the bottom of the pile $(5.19 \pm 2.12)$.

A rather abysmal score was also seen when evaluating the current scenario of faculty openings in medical colleges across India. The participants could only muster a score of $4.85 \pm 1.96$.

Lastly, when enquired about their overall satisfaction with MD Pharmacology, we obtained a cautious yet positive response of $6.38 \pm 1.39$.

\section{DISCUSSION}

The field of pharmacology is witnessing a revolution in our times. The scope of pharmacology as a subject has risen from being merely a theoretical one to being a bridge between knowledge and application of the clinical decision making process. As per the rise in expectations from a pharmacologist, however there is observed a visible lacuna in their preparatory standards.

In recent years, owing to the revised MCI guidelines on reduction of faculty requirement in medical colleges and overall slump in clinical trial conduction due to a plethora of reasons, the future of PG residents in Pharmacology is not as lucrative as it once was. ${ }^{5}$

In our study we aim to assess the views of PG residents on various topics holding immense relevance for their career choices. The aim of our study is not to contradict the current scenario or teaching practices, but rather to initiate a thought-provoking process to better gauge the reality facing us.

From the results obtained from our study, we infer that PG residents have a fair understanding of the current regulations surrounding $\mathrm{AE}(6.14 \pm 1.53)$ and most of them had a positive outlook for $A E$ as per its relevance in $P G$ research/industry level $(6.34 \pm 2.73)$ and $P G$ academic level $(5.95 \pm 2.03)$. This finding of ours is complimentary to the study conducted by Shehnaz et al which evaluated the faculty view on $\mathrm{AE}$ and reported that an overwhelming majority of their respondents $(75 \%)$ were aware of the advantages of $\mathrm{AE}$ in the application of practical pharmacology. ${ }^{6}$

The role of $\mathrm{AE}$ in providing valuable pre-clinical safety and efficacy data, which in turn is one of the necessities for submission to drug regulatory authorities for furthering studies in humans, is irrefutable. ${ }^{7}$ Thus we infer that $\mathrm{AE}$ is an indispensible aspect of pharmacology which is a necessity for the advent of a complete pharmacologist.

With an ever more increasing emphasis (both by MCI and CPCSEA) on alternative techniques to animal experimentation, this is one query which needs to be answered in totality. From our survey we found that only $42.5 \%(n=20)$ of the repliers had access to alternative techniques such as computer simulators at their college/institution level. Also we see that the application, availability of these alternatives and their emphasis by the university/college is still abysmally low.

The MCI amendment in 2009 asked all medical colleges to use alternatives to animal experiments in the undergraduate medical course. ${ }^{8}$ At the postgraduate level however, a dearth of clear set guidelines is a cause of worry for both the students and the teachers. Across the entire country, the syllabus of MD pharmacology has $\mathrm{AE}$ 
as a major component but the current rules and guidelines on their role are unclear and downright confusing.

From our study we also establish that a majority of the students $(\sim 80 \%)$ want either a subsidized availability of the alternatives or a procedure involving once only payment. The limited budget of most medical colleges does not fully justify expensive yearly subscription renewals.

Most of the participants rated that they were adequately prepared by their current teaching curriculum for teaching jobs in medical colleges $(6.57 \pm 1.49)$. However, when enquired about same for preparedness in the pharmaceutical industry, an overall low rating was observed (4.25 \pm 2.03$)$

When enquired about the desirable changes in PG teaching programs, the participants were of an opinion to inculcate compulsory pharmaceutical industry rotation $(31.9 \%)$ and to make the syllabus more clinical pharmacology oriented $(29.7 \%)$. This finding is in concordance with the study conducted by Chakraborty which also mentions internship opportunities as the need of the hour. ${ }^{9}$

Ghosh et al in their evaluation state that the postgraduate pharmacology curriculum should incorporate some advanced modules on clinical trial conduct and management. ${ }^{10}$ This inference of theirs is well accepted by the current crop of budding pharmacologists as per our study.

Clinical rotations were also held in high regard as per our study (6.74 \pm 1.68$)$, thus establishing that the current crop of budding pharmacologists is well aware of the advantages of patient centric teaching. The medical council of India has time and again emphasized the integration of teaching of pharmacology with internal medicine. ${ }^{11}$ Avenues of rational pharmacotherapeutics, management of adverse drug reactions, as well as integrated learning with other clinicians are some of the aspects which are at best dealt in real world clinical scenarios.

The importance of PG seminars and clinical case discussions in the PG teaching curriculum is also held in high esteem as reflected by the results of our study $(7.21 \pm 1.70$ and $7.06 \pm 2.26$ respectively). Badyal et al also quote the importance of these methods as predominant teaching aids for a PG resident undergoing training in pharmacology. 5

Good quality and scientifically relevant research is the back bone of medical science. Biomedical research though necessary as dissertation etc. has now been reduced to the status of a mere formality for examination eligibility in a majority of the institutes. The participants also opined that the general outlook towards meaningful research was less than satisfactory $(4 \pm 2.6)$. This should serve as a wake-up call for all concerned institutions to lay foremost emphasis on research based training for the betterment and advancement of medical science.

As per (Figure 7) of our study, it is revealed that the participants had a relatively obscure idea about the responsibilities of a regulator in the pharmaceutical industry setting $(36.1 \%)$. The role of regulator is shrouded in somewhat secrecy and lacks transparency when compared with other sub-disciplines within the pharmaceutical industry. A suggestion to this regard is once again the need for industrial internships to experience first-hand know how process, as they open channels not dealt with in text books. The responsibilities of a medical advisor, clinical pharmacologist and clinical trial co-ordinator were relatively well appreciated.

As per our study, the participants realized the importance of GCP/GLP certification, industrial internships and paper publication as methods to boost their curriculum-vitae. These tools are of utmost importance as they form the basis for various practices within the pharmaceutical industry. MBA was a skill which the participants deemed comparatively less important $(4.72 \pm 2.3)$, probably due to its association with only the higher echelon of management and marketing side of job profile and with not much relevance to the other sub-disciplines within the pharmaceutical industry.

One rather surprising finding of our study was that almost $50 \%$ of the PG residents pursuing MD in pharmacology were unable to differentiate their role and hierarchy in the pharmaceutical industry from $\mathrm{PhD}$, Pharm $\mathrm{D}$ and $\mathrm{M}$ Pharm graduates. This chaotic situation is a pressing matter for all MD pharmacology pursuers as often job listings of pharmaceutical companies also sometimes fails to offer specific eligibility criteria for different roles in the pharmaceutical industry. Thus specific minimum eligibility guidelines and role definition are a need of the hour.

MD Pharmacology, being a PG training program needs to provide hands-on practical experience in the pharmaceutical industry and clinical trials setting. The skills learnt from such a practical approach will benefit the field of healthcare and thus the society as a whole. Kshirsagar et al in their study highlight how with the utilization of training programs and workshops, talks by role models and subject experts, sessions on ethics etc., a need for current and future demands on this aspect of pharmacology can be accurately decreed. ${ }^{12}$

Clinical trials are the most widely sought after subdiscipline within the pharmaceutical industry (7.06 \pm 1.67$)$, while attraction level of BA/BE studies was the minimum (5.21 \pm 1.41$)$. This rather small disparity might be attributed to the more glamorous presentation of clinical trials with the rather mundane domain of BA/BE studies. 
(Figure 10) of our study clearly establishes the superiority of Pharma tech workshop (conducted by KEM Mumbai) as per the reviews of PG residents based on the usefulness for preparation for the pharmaceutical industry (7.06 \pm 2.28$)$. The industry-centric approach along with the on-site visits etc. make this as the most sought after workshop for candidates aiming to pursue a career in the pharmaceutical industry.

Lastly, when asked about their overall satisfaction with MD pharmacology as a career choice, the response was ascertained as positively cautious $(6.38 \pm 1.39)$. The dynamism of the field of pharmacology along with its ever increasing importance in bed-side medicine has its well defined attributes and gently nudges the PG resident to be optimistic about the future. The challenges faced by the pharmacologist are aplenty, but it is up to us to devise ways to meet and tackle them head on.

A limitation of this study was a sample-size of $n=47$ is relatively small, thus a larger study can be planned on the footprints of this study to address this shortcoming.

\section{CONCLUSION}

The changes ushered by the advent of numerous pharmaceutical and research based industries has led to a conscious re-thinking pertaining to the validity of PG curriculum. Evaluation of practices of animal experimentation, evolution of post graduate teaching curriculum and preparedness for the pharmaceutical industry are some of the dilemmas being faced by a post graduate resident.

From our study we conclude that the importance of AE in pharmacology is irrefutable, although advent of alternatives is the direction to be headed in. The current syllabus is found wanting and archaic at times, thus warranting changes such as inculcation of industrialinternships and greater emphasis on bed-side teaching and clinical pharmacology. The preparedness for pharmaceutical industry is also deduced to be rather low and work needs to be done to inculcate a culture of teaching based on research and technology.
Funding: No funding sources Conflict of interest: None declared

Ethical approval: The study was approved by the Institutional Ethics Committee

\section{REFERENCES}

1. Dikshit RK. Postgraduate education in medical pharmacology. Indian J Pharmacol. 2007;39:171.

2. Shivprakash. Pharmacology: taking it forward. Indian J Pharmacol. 2007;39:3-4.

3. Gupta V. Postgraduate education in medical pharmacology: A student's viewpoint. Indian J Pharmacol. 2007;39:256.

4. Dang A, Garg A, Rataboli PV. Current perspectives of medical pharmacology post graduates in India: Need to commute the system. J Clin Diag Res. 2009;3:1601-2.

5. Badyal DK, Desai C, Tripathi SK, Dhaneria SP, Chandy SJ, Bezbaruah BK. Postgraduate pharmacology curriculum in medical institutions in India: Time for need-based appraisal and modifications. Indian J Pharmacol. 2014;46(6):584-9.

6. Shehnaz SI, Agarwal AK, Arifulla M. Faculty outlook toward animal experiments in post-graduate medical education. J Young Pharm. 2013;5(1):32-4.

7. Arora T, Mehta AK, Joshi V, Mehta KD, Rathor N, Mediratta PK, et al. Substitute of animals in drug research: an approach towards fulfilment of 4R's. Indian J Pharm Sci. 2011;73:1-6.

8. Badyal DK, Desai C. Animal use in pharmacology education and research: The changing scenario. Indian J Pharmacol. 2014;46(3):257-65.

9. Chakraborty A. A survey on postgraduate pharmacology education in India. Indian J Pharmacol. 2010;42(4):252-6.

10. Ghosh RK, Ghosh SM, Datta S. Training of postgraduate pharmacologists in India - The need for alignment with the emerging roles in the pharmaceutical industry. J Postgrad Med. 2010;56:168-9.

11. Kela AK, Mehta VL. Impact of inclusion of clinical projects in undergraduate teaching. Indian $\mathrm{J}$ Pharmacol. 1993;25:249-50.

12. Kshirsagar NA, Bachhav SS, Kulkarni LA, Kumar V. Clinical pharmacology training in India: Status and need. Indian J Pharmacol. 2013;45(5):429-33.

Cite this article as: Dass $\mathrm{P}$, Javedar P, Mishra R, Gulabani M. Post graduate survey and evaluation of role of animal experimentation, status and relevance of current syllabus, and challenges for alignment with requirements of the pharmaceutical industry. Int J Basic Clin Pharmacol 2016;5:935-41. 


\section{APPENDIX 1}

\section{QUESTIONNAIRE}

\section{Post graduate survey of role of animal experimentation, relevance of current syllabus and challenges in pharmaceutical industry}

Please answer the questions on a scale of 0 to 10 with 0 being the least and 10 being the maximum possible value, with 5 being neutral.

1. Evaluate your awareness regarding current MCI/ University guidelines on animal experimentation in MD Pharmacology at postgraduate level.

2. Give your opinion about relevance of animal experiments
a. At undergraduate level
b. At postgraduate teaching level
c. At postgraduate research/industry level

3. In your opinion what type of animal experimentations can be excluded? (Tick one)

a. Those involving animal sacrifice.

b. Any kind of animal experimentation.

4. Rate your knowledge about the available alternatives to animal experimentation.

5. Rate the availability of these techniques

6. Mention the alternative techniques you have access to at your institution.

7. Evaluate the application of these alternatives at your university/college level.

8. What is your preference relating to pricing of these alternative techniques? (Tick One)
a. Once payment
b. Yearly subscription
c. Govt./university sponsored

9. Rate how well your postgraduate curriculum equips you for the challenges ahead?

a. For teaching in medical colleges

b. For pharmaceutical industry

10. What improvements can be inculcated in the PG syllabus? (Tick most relevant choice)
a. Revision of entire syllabus
b. Additional emphasis on clinical pharmacology
c. Compulsory industry rotation
d. Patient care and interaction

11. Evaluate your purview on the necessity of clinical rotations.

12. Evaluate the importance of the following from your post graduate curriculum:
a. Seminars
b. Journal club
c. Pedagogy

d. Protocol writing

e. Clinical case discussion

f. Experiments

g. Short techniques

13. Rate the encouragement level in your institute/department towards research.

14. Answer in yes or no if you are aware about your responsibilities in the pharmaceutical industry as a:
a. Medical advisor.
b. Regulator
c. Researcher
d. Clinical trial coordinator
e. Clinical pharmacologist

15. Rate these add-ons based on their usefulness in the pharmaceutical industry:
a. GCP/GLP certification
b. MBA
c. Industrial internships
d. Paper publications

16. Do you clearly understand and differentiate your role and hierarchy in the pharmaceutical industry when compared with $\mathrm{PhD}$, PharmD, and MPharm graduates? (Yes/No)

17. Evaluate the following positions based on their attraction:
a. Medico marketing/sales
b. Basic research
c. BA/BE studies
d. Clinical trials

18. Rank the following conferences/workshops related to their usefulness for preparation of postgraduate students for the pharmaceutical industry
a. IPSCON
b. PHARMATECH (KEM Mumbai)
c. NWCPT (PGI Chandigarh)
d. Pharmacogenomics workshop (JIPMER Puducherry)
e. ISRPTCON

19. Rate the current scenario of faculty positions in medical colleges across India.

20. Sum up your overall satisfaction with MD Pharmacology. 